\title{
Carbon Fiber TOW Angle Determination using Microwave Reflectometry
}

\author{
William C. Wilson, Jason P. Moore, Peter D. Juarez \\ Nondestructive Evaluation Sciences Branch \\ NASA Langley Research Center \\ Hampton, VA, USA \\ William.C.Wilson@nasa.gov
}

\begin{abstract}
NASA's Advanced Composites Project is investigating technologies that increase automated remote inspection of aircraft composite structures. Therefore, microwave Frequency Domain Reflectometry (FDR) is being investigated as a method of enabling rapid remote inspection of angular orientation of the tow using microwave radiation. This work will present preliminary data demonstrating that frequency shifts in the reflection spectrum of a carbon fiber tow sample are indicative of the angle of the tow with respect to an interrogating antenna's linear polarized output.
\end{abstract}

Keywords- Microwave; frequency domain reflectometry; electromagnetic; carbon fiber; tow; orientation

\section{INTRODUCTION}

NASA's Advanced Composites Project (ACP), part of the Advanced Air Vehicles Program (AAVP) is investigating technologies that increase aircraft component inspection throughput by $30 \%$ through the development of quantitative and practical inspection methods for both in-service and in-process applications [1]. Microwave Frequency Domain Reflectometry (FDR) is being investigated as a method of enabling detection of tow misalignment during layup while structures are being fabricated through the use of Radio Frequency (RF) ElectroMagnetic (EM) radiation.

The goal of this work is to investigate the use of RF energy to remotely (non-contact) determine the angle of carbon fiber tow while it is being laid down during the fabrication process. One potential application for this technology is monitoring the carbon fiber tow as it is being placed by a fabrication robot such as NASA's carbon fiber fabrication robot ISAAC (Integrated Structural Assembly of Advanced Composites). For this application the antenna would be attached on the robotic fabrication head in close proximity to the tow, but remotely interrogating the tow as it is being positioned. We are investigating near field placement, however, if necessary the antenna could be place in the farfield, since others have used this technique to interrogate glass fiber reinforced polymer (GFRP) on concrete structures [2].

For this work, carbon fiber orientation is being investigated. The anisotropy of the tow material properties causes changes in the reflection coefficient or scattering parameter $\left(\mathrm{S}_{11}\right)$ response due to fiber orientation. The "microwave signal is strongly influenced by the relative orientation of the fibers and the microwave signal polarization" [3]. The reflection will be at a maximum when the antenna polarization direction is parallel to the fiber direction [4]. Microwave energy has been used in a similar manner to determine the angle of the grain of wood [5]. Microwaves have also been used in the past to determine the fiber angle within unidirectional carbon fiber reinforced polymer (CFRP) [6]. Although others have used microwaves to investigate the angle of carbon fibers, none have used this technique for measuring tows during the manufacturing process.

\section{EXPERIMENTAL SETUP}

The test rig was constructed of an aluminum turn table torus with a plastic $360^{\circ}$ protractor for accurate angular positioning of the tow sample. A $6.4 \mathrm{~mm}$ wide, $250 \mathrm{~mm}$ long IM7 tow is attached to the rotation ring using Kapton tape. The test rig holds the tow in a planar position during rotation. The antenna remains in a fixed position $120 \mathrm{~mm}$ above the test rig while the tow is rotated beneath it for measurements. The tests begin with the tow aligned to zero degrees with respect to the transverse magnetic (TM) orientation where the maximum amplitude response is expected (Fig 1.). When the rotation angle is $90^{\circ}$ with respect to the TM (perpendicular) the tow is parallel to the transverse electric (TE) orientation at the antenna. The EM signature of the carbon fiber tow is interrogated using an Agilent N5230C vector network analyzer connected to an A. H. Systems SAS-571, $700 \mathrm{MHz} \sim 18 \mathrm{GHz}$, linearly polarized, dual ridge horn antenna. The antenna is

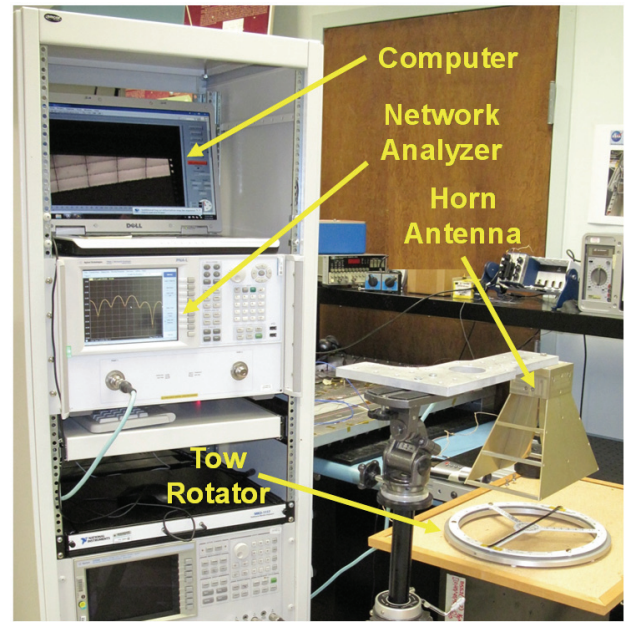

Fig. 1. Experimental setup: network analyzer, computer, tow rotator rig and horn antenna. 


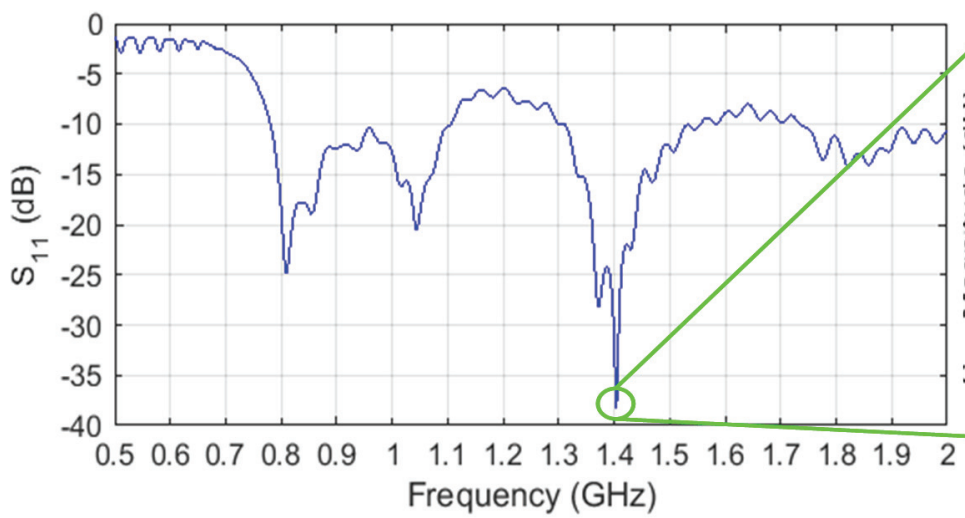

(a)

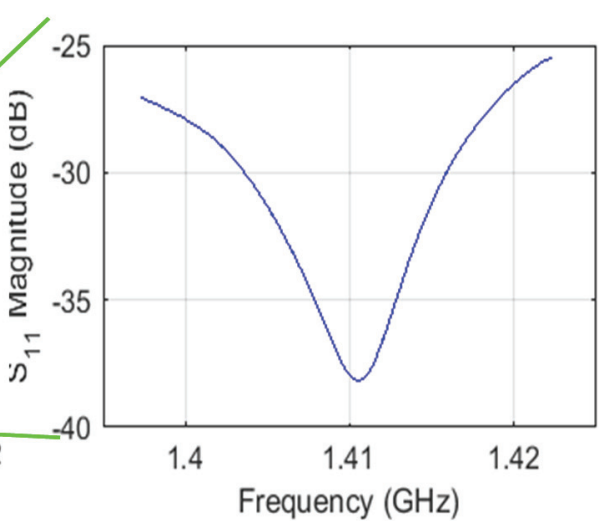

(b)

Fig. 2. (a) The EM signature $\left(\mathrm{S}_{11}\right.$ response) of the tow from $0.5 \mathrm{GHz}$ to $2 \mathrm{GHz}$. (b) The $\mathrm{S}_{11}$ response for the $25 \mathrm{MHz}$ bandwidth used for this work.

located $120 \mathrm{~mm}$ above the tow. The network analyzer is connected to the computer via a USB cable and the data is collected and stored using LabVIEW on the computer.

The system is a microwave reflectometer so a single antenna is used for both sending and receiving the RF energy. The network analyzer outputs a $25 \mathrm{MHz}$ bandwidth chirp signal from $1.3973 \mathrm{GHz}$ to $1.4223 \mathrm{GHz}$, and then it captures the returned $\mathrm{S}_{11}$ reflection parameters. By tracking frequency shifts in the $\mathrm{S}_{11}$ response, angular orientation measurements can be achieved.

Before taking data, the basic $\mathrm{S}_{11}$ response of the tow was measured from $500 \mathrm{MHz}$ to $2 \mathrm{GHz}$ (Fig. 2 (a)). The complex $\mathrm{S}_{11}$ response across that band shows many peaks and troughs in amplitude. Because the troughs have a more narrow response than peaks, the trough with the lowest amplitude and greatest amplitude change was chosen for this work, Fig. 2 (b). The frequency band of $1.3973 \mathrm{GHz}$ to $1.4223 \mathrm{GHz}(25 \mathrm{MHz}$ bandwidth) was selected.

\section{RESULTS}

After determining the EM signature and which frequency chirp range to utilize, measurements were taken without the tow present on the test rig. The tow angle is first aligned to $0^{\circ}$ with respect to the TM orientation of the antenna. The test rig is then rotated from $0^{\circ}$ to $180^{\circ}$ in $10^{\circ}$ steps. At each rotation step 24 data sets are taken (Fig. 3).

The reflectance is expected to have a maximum at $0^{\circ}$ and $180^{\circ}$ when the test rig is aligned parallel with the antenna TM direction, and a minimums at $90^{\circ}$ when the test rig is perpendicular to the antenna TE direction. The frequency shifts from the test rig without a tow present do not follow the shifts that would be expected from angular changes. The unexpected shifts may be a result of varying room temperature and humidity throughout the experiment. The frequency shifts for a change from $0^{\circ}$ to $90^{\circ}$ of rotation, are relatively small $(+30$ to $-50 \mathrm{KHz})$ when compared to $\sim 7 \mathrm{MHz}$ which is the value measured with a tow present. Therefore, $\pm 50 \mathrm{KHz}$ will be used as the noise floor for further tow angle experiments.

For the next experiment the tow was attached to the rotation ring using Kapton tape. The tow angle is first at $0^{\circ}$ alignment with the TM orientation of the antenna. The tow is then rotated from $0^{\circ}$ to $360^{\circ}$ in $10^{\circ}$ steps. At each rotation step 24 data sets are taken (Fig. 4). The reflectance is expected to have

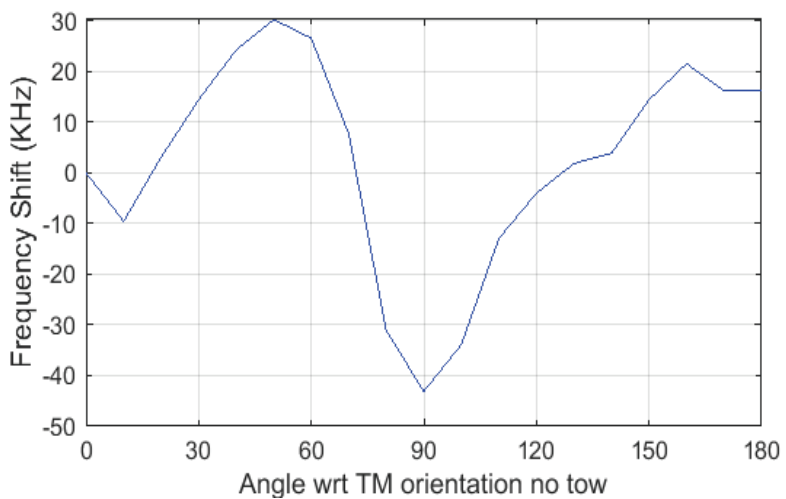

Fig. 3. The average $S_{11}$ frequency shift vs angle between the test rig and the TM axis of the horn antenna without any tow.

maximums at $0^{\circ}, 180^{\circ}$ and $360^{\circ}$ degrees when the tow is aligned parallel with the antenna TM direction, and to have minimums at $90^{\circ}$ and $270^{\circ}$ when the tow is perpendicular to the antenna TM direction. The points in Fig. 4 do not correspond very well to the expected cosine wave. However, the network analyzer records the real and imaginary components of the $S_{11}$ response. In addition to the magnitude of the $\mathrm{S}_{11}$ response, the data can be used to calculate the phase, frequency shift, impedance, and resistance values. All of these parameters were investigated, however, the best results were obtained by using the centroid of the amplitude signal to calculate the frequency shift (Fig. 5).

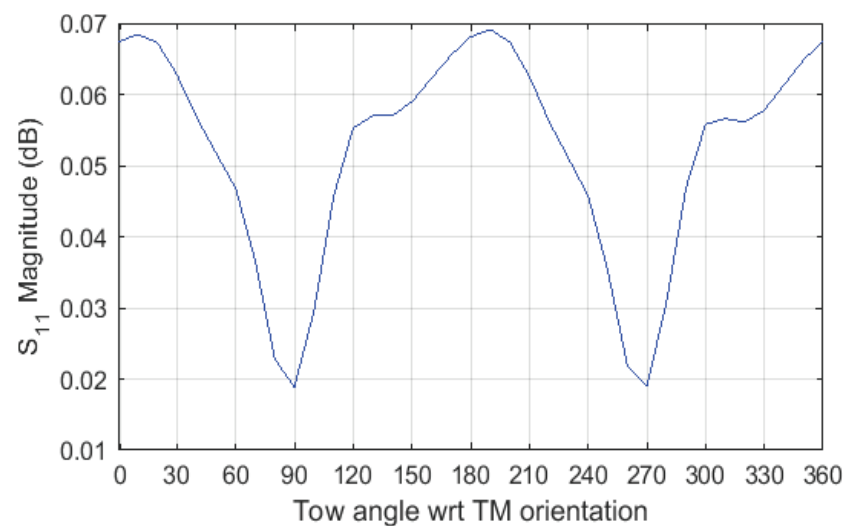

Fig. 4. The averaged $S_{11}$ magnitude as the angle between the test rig and the alignment of the TM axis of the horn antenna is varied. 


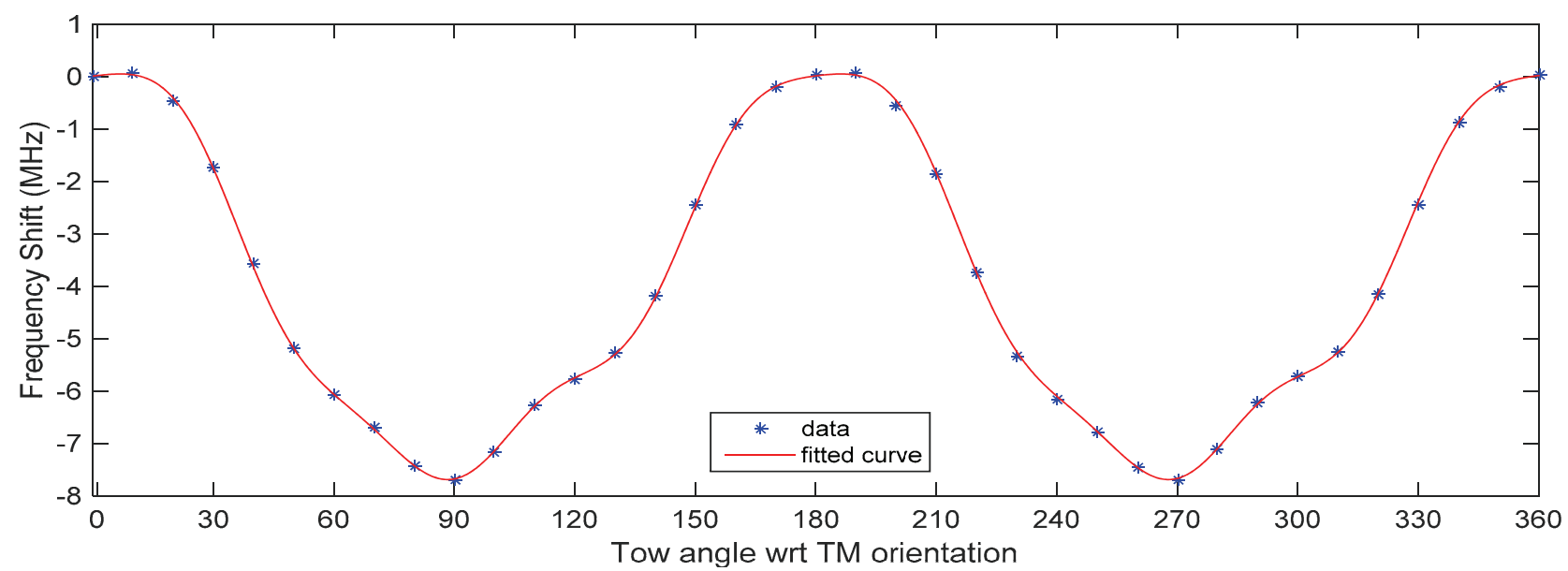

Fig. 5. The averaged $S_{11}$ response (frequency shift) vs angle between the tow and the TM axis of the horn antenna.

The blue asterisks in Fig. 5 are averaged values of the 24 frequency shift values calculated from the centroid data at each angular measurement. The measured frequency shift is proportional to the reflectance; therefore it has maximums at $0^{\circ}$, $180^{\circ}$ and $360^{\circ}$ degrees and minimums at $90^{\circ}$ and $270^{\circ}$. The shape of the plot in Fig. 5 is closer to that of a cosine wave when compared to Fig. 4. The change in the signal frequency response shows a strong dependence on the angle of the tow; therefore the frequency shift of the $S_{11}$ response may be used for tow orientation sensing for most tow angles $\left(20^{\circ}-80^{\circ}\right.$ and $110^{\circ}-160^{\circ}$, for example).

To determine the resolution of the system, the average frequency shift for the 24 points, from $10^{\circ}$ to $90^{\circ}(7.7561 \mathrm{MHz})$ was dived by the angular change $\left(80^{\circ}\right)$. The result is a resolution of $96951 \mathrm{~Hz} /$ degree, and the signal to noise ratio (SNR) was calculated to be 64.63 .

To capture the shape of the frequency shift as the angle changes a fourth order Fourier fit to the data was performed using:

$$
\begin{aligned}
f(x)= & a_{0}+a_{1} \cos (x w)+b_{1} \sin (x w)+a_{2} \cos (2 x w)+b_{2} \sin (2 x w)+ \\
& a_{3} \cos (3 x w)+b_{3} \sin (3 x w)+a_{3} \cos (4 x w)+b_{3} \sin (4 x w) .
\end{aligned}
$$

The fit is plotted in red in Fig. 5 and the coefficients for the fit are given in Table 1. The coefficients are in MHz, except for " $w$ " which is in inverse degrees.

TABLE I. TABLE 1 FOURTH ORDER FOURIER FIT COEFFICIENTS.

\begin{tabular}{|l|r|l|r|}
\hline$a_{0}$ & -3.956 & $w$ & 0.03501 \\
\hline$a_{1}$ & 3.816 & $b_{1}$ & 0.1562 \\
\hline$a_{2}$ & 0.4289 & $b_{2}$ & 0.2704 \\
\hline$a_{3}$ & 0.02951 & $b_{3}$ & -0.06143 \\
\hline$a_{4}$ & -0.3012 & $b_{4}$ & -0.05304 \\
\hline
\end{tabular}

The fit is presented to give a visual indication of the changes in the $\mathrm{S}_{11}$ response as the angle changes. Future work will determine the physical phenomena that correctly captures this behavior. Also, a sinusoidal dependence was expected, so further investigation is needed to optimize the test setup and to include post processing to determine if better results can be achieved.

\section{CONCLUSION}

In this preliminary work, a microwave reflectometer has been used to demonstrate remote sensing of the angular orientation of a carbon fiber tow sample using frequency shifts in the $S_{11}$ response. An average sensitivity of $96951 \mathrm{~Hz} /$ degree resolution has been measured; however the dependence is strongly sinusoidal. A noise floor of $\pm 50 \mathrm{KHz}$ was measured without the tow present and the signal to noise ratio (SNR) was calculated to be 64.63. This work supports an ongoing project to automate aircraft composite inspections during the manufacturing process.

Further optimization will be necessary to increase the angular resolution of the system. A smaller antenna with lower wavelength should allow for higher resolution. Other areas of investigation include, post processing to reduce noise and alternate interrogation frequencies. Future work will also investigate the largest distance between the tow and the antenna to determine the best placement of the antenna on the robot fabrication head.

\section{REFERENCES}

[1] Techport, "Advanced Composites Project, Advanced Air Vehicles Program, Aeronautics Research Mission Directorate (ARMD)," NASA Langley Research Center, Project Status Report, URL: https://techport.nasa.gov/view/13280, 2016, p. 9.

[2] T.-Y. Yu and O. Büyüköztürk, "A Far-Field Airborne Radar NDT Technique for Detecting Debonding in GFRP-Retrofitted Concrete Structures," NDT \& E International, vol. 41, no. 1, pp. 10-24, 2008.

[3] S. Kharkovsky, A. C. Ryley, et al., "Dual-Polarized Microwave NearField Reflectometer for Non-Invasive Inspection of Carbon Fiber Reinforced Polymer (CFRP) Strengthened Structures," in Proc. 2006 IEEE Instrumentation and Measurement Technology Conference Proceedings, Sorrento, Italy, April 24-27, 2006, pp. 2108-2111.

[4] S. Kharkovsky, A. C. Ryley, et al., "Dual-Polarized Near-Field Microwave Reflectometer for Noninvasive Inspection of Carbon Fiber Reinforced Polymer-Strengthened Structures," Instrumentation and Measurement, IEEE Transactions on, vol. 57, no. 1, pp. 168-175, 2008.

[5] S. A. Malik, D. K. Ghodgaonkar, et al., "Measurement of Wood Grain Angle using Free-Space Microwave Measurement System in 8-12 GHz Frequency Range," in Proc. Asian Conf. on Sensors and the International Conf. on new Techniques in Pharmaceutical and Biomedical Research, Kuala Lampur, Maylasia, Sept. 5-7, 2005, pp. 213-218.

[6] K. Urabe, "Rotative Polarization System of Millimetric Wave for Detecting Fiber Orientation in CFRP," Journal of reinforced plastics and composites, vol. 11, no. 2, pp. 179-197, 1992. 